\title{
Anthropologie de la littérature, pratiques symboliques et diabolie critique
}

\section{Hélène Merlin-Kajman}

\section{(2) OpenEdition}

\section{Journals}

Édition électronique

URL : http://journals.openedition.org/edl/830

DOI : $10.4000 /$ edl. 830

ISSN : 2296-5084

\section{Éditeur}

Université de Lausanne

\section{Édition imprimée}

Date de publication : 15 mai 2015

Pagination : 115-142

ISBN : 978-2-940331-45-1

ISSN : 0014-2026

\section{Référence électronique}

Hélène Merlin-Kajman, « Anthropologie de la littérature, pratiques symboliques et diabolie critique », Études de lettres [En ligne], 1-2 | 2015, mis en ligne le 15 mai 2018, consulté le 18 décembre 2020 URL : http://journals.openedition.org/edl/830 ; DOI : https://doi.org/10.4000/edl.830 


\section{ANTHROPOLOGIE DE LA LITTÉRATURE, PRATIQUES SYMBOLIQUES ET DIABOLIE CRITIQUE}

Cet article reparcourt l'œuvre de Claude Reichler en montrant l'importance de ses analyses des "modélisations symboliques", non seulement pour l'analyse des textes littéraires, mais aussi pour notre réflexion sur le discours critique et même sur l'enseignement de la littérature. Deux questions forment le fil rouge de la réflexion: existe-t-il une lecture "diabolique» d'un texte littéraire, une pratique "diabolique» de nos disciplines? Comment l'absence d'une description des Alpes dans les Mémoires d'un auteur du XVII e siècle amené à les traverser se trouve éclairée, ana-chroniquement mais sans contresens historique, par l'analyse, elle-même symbolique (et jamais "diabolique») du "paysage alpin» effectuée par Claude Reichler?

C'est avec émotion que je prends la parole devant vous ${ }^{1}$ pour vous présenter quelques réflexions concernant les enjeux d'une possible définition anthropologique de la littérature, définition régulièrement évoquée par Claude Reichler dans de nombreux textes, et tout particulièrement élaborée dans "La littérature comme interprétation symbolique». Cet article très dense est paru en 1989 dans L'interprétation des textes, ouvrage collectif qu'il avait dirigé et qui fournit le point de départ à notre colloque. Dans son "Avant-propos», Claude Reichler résumait sa propre contribution en disant:

I. Cet article a d'abord pris la forme d'une communication lors du colloque organisé par l'Université de Lausanne en hommage à quatre de ses professeurs qui partaient à la retraite, dont le professeur Claude Reichler. Son trajet théorique étant complètement lié à la relecture, à cette occasion, de l'œuvre de Claude Reichler, c'est-à-dire au trouble et à l'admiration actuels causés par cette re-lecture, j'ai désiré ne pas supprimer les marques orales de mon article qui doit tout à cette occasion. 
Pour ma part, j'invoque une "définition anthropologique de la littérature" qui permettrait de mettre en lumière la place occupée par celleci dans l'ensemble des représentations formant lien entre les individus et le groupe ${ }^{2}$.

Dans ces années-là, pour ce qui me concerne, je ne pouvais guère entendre ce projet dans toute sa force, tout simplement parce que, marquée par Michel Foucault, venue du marxisme, du structuralisme et, plus discrètement, de la psychanalyse, j'étais alors totalement indifférente aux enjeux anthropologiques de nos objets «littéraires». Je crois cependant qu'il n'a rien perdu de son actualité, et même, aussi paradoxal que cela puisse paraittre, de son "urgence».

Mais dire cela, c'est préciser un peu la nature de mon émotion. Elle est bien sûr liée à la circonstance, celle du départ à la retraite de Claude Reichler, professeur de littérature française de renom international, très grand chercheur, et ami de longue date de surcroît. Pour l'anecdote, j’ai écouté Claude Reichler pour la première fois dans le cadre d'une série de rencontres organisées à l'EHESS par Christian Jouhaud et Roger Chartier. C'était en 1990. Claude Reichler a présenté ce jour-là une analyse lumineuse de stances théâtrales du XVII e siècle pour montrer «la nature symbolique du lien entre une forme littéraire et son contexte» ${ }^{3}$. Sa démarche restait très près de ce que nous avons coutume d'appeler la littérarité du texte, dégageant ses figures, ses effets de rupture dans l'économie discursive et dramatique générale de l'œuvre théâtrale. Mais elle ajoutait une perspective "symbolique» à la simple considération de la littérarité: elle montrait qu'une forme aussi particulière, isolée, forme d'une "grande intensité ornementale»" ${ }^{4}$, ce chant de personnages emprisonnés et près de mourir comme Sénèque dans La mort de Sénèque de Tristan L'Hermite, trouvait sa motivation symbolique indirecte dans des contraintes socio-politiques extérieures diverses, par exemple «l'asservissement des écrivains à l'éloge du roi " 5 , mais elles-mêmes modélisées selon le schéma du divorce des bellatores et des oratores. Selon Claude Reichler,

\footnotetext{
2. C. Reichler, «Avant-propos», p. 5.

3. C. Reichler, «Les stances devant la mort dans le théâtre baroque ou le retrait du sage», p. 79.

4. Ibid., p. 90.

5. Ibid., p. 91.
} 
les stances constituent un «outil interprétatif capable de faire apparaître» qu' «une même modélisation symbolique, venue d'une mutation historique profonde, gouverne ces manifestations divergentes" ${ }^{6}$.

Pour la jeune chercheuse que j'étais alors, l'exposé de Claude Reichler, devant des historiens avec lesquels j'allais dialoguer pendant quelques années, constituait une formidable preuve qu'on pouvait sortir de l'alternative de la théorie de l'autonomie du texte littéraire et de la théorie contraire selon laquelle sa signification et sa fonction s'épuiseraient dans son contexte, non moins du reste que de la théorie, liée à la précédente mais plus sophistiquée du point de vue de ses outils d'analyse, selon laquelle le texte littéraire ne serait qu'un discours requérant une analyse rhétorique ou pragmatique adéquate ${ }^{7}$. Comme l'écrit Claude Reichler dans "La littérature comme interprétation symbolique», "la littérature existe autrement que comme document ou comme jeu de formes» ${ }^{8}$; et il va même jusqu'à affirmer, proposition plus difficile, qu'elle n'est pas exactement de l'ordre de la représentation (je dirais a fortiori qu'elle n'est pas de l'ordre du discours). Il n'y a littérature que lorsqu'un texte offre au lecteur une interprétation "symbolique", et non pas "analytique", des schèmes générateurs de représentations, schèmes que Claude Reichler appelle des modélisations. Ceci situe le texte littéraire entre le mythe et la névrose, deux constructions constituant elles aussi des interprétations symboliques des modélisations du monde: mais contrairement au mythe, le texte littéraire est une interprétation symbolique individuelle, comme la névrose; et contrairement à cette dernière, le texte littéraire présente une interprétation symbolique conciliable avec les modélisations partagées par tous, comme le mythe.

Ces définitions de Claude Reichler ouvrent des perspectives immenses à l'analyse littéraire. Mais pour le comprendre, il m'aura fallu un long détour. C'est bien pour échapper aux mêmes alternatives - structure formelle close sur elle-même, objet socio-symbolique, discours, document... - que j'avais choisi pour sujet de thèse la notion de "public"

\section{Ibid.}

7. Sur ce que Claire Badiou-Monferran a appelé «le moment discursiviste», cf. sa remarquable introduction aux actes d'un colloque qu'elle avait organisé, en réunissant des littéraires et des linguistes autour d'un corpus abordé par les uns et les autres sans qu'ils se soient jamais lus jusqu'à cette rencontre (C. Badiou-Monferran, "Présentation: Le dialogue interdisciplinaire»).

8. C. Reichler, « La littérature comme interprétation symbolique», p. 109. 
au XVII ${ }^{e}$ siècle. Ce choix impliquait en effet le rejet du concept de "texte» - mais également, dans une certaine mesure, le rejet de celui de «contexte». C'est la présupposition de leur articulation - présupposition qui s'appelle souvent, mais pas toujours, le "public»; présupposition sans laquelle rien n'aurait lieu, tout simplement - qui m'intéressait, et m'intéresse toujours. Pour le dire autrement, je n'ai, quant à moi, jamais travaillé sur un texte littéraire sans partir soit d'une réception attestée, soit de son geste de destination ${ }^{9}$; et même, si possible, du trajet complet de transmission d'un texte. J'entends par «trajet» non pas tant son trajet discursif, social, matériel, que le dispositif imaginaire et symbolique qui noue un geste de destination à une scène de réception. En un sens, la "théorisation" du texte ne m’a jamais intéressée. Mais à force de l'observer - lui, le texte - à partir de ces dispositifs, j'ai fini par porter de l'attention aux «représentations formant lien entre les individus et le groupe » ${ }^{10}$, donc à l'efficacité symbolique.

C'est là que j'ai rejoint, que je rejoins, avec un train de retard, les préoccupations anthropologiques de Claude Reichler. D'où une autre cause à mon émotion: repartir de l'œuvre de Claude Reichler pour réfléchir sur notre façon de définir le texte - le texte littéraire - et même plus précisément sur nos raisons de qualifier un texte de «littéraire»-, c'est pour moi aussi prendre la mesure du trajet parcouru, et du trajet qui reste à parcourir. Bref, je me sens honorée, intimidée, mais enthousiaste, de la chance qui m'est ainsi offerte d'avoir à mettre ma propre réflexion à l'épreuve de celle d'un aussi grand théoricien de la littérature et des phénomènes symboliques que Claude Reichler.

Relire ou lire l'œuvre de Claude Reichler m'a en effet saisie, ou plutôt ressaisie, d'admiration: la beauté des lectures d'un côté, la richesse, l'ampleur et la cohérence des propositions théoriques de l'autre, s'accompagnent de l'extraordinaire effet de présence, toute en discrétion volontaire, d'un sujet engagé dans sa recherche et qui cherche à soutenir cet engagement avec une exacte justesse. Cette qualité est suffisamment rare pour que je la souligne avec gratitude.

La diabolie, notamment, paru chez Minuit en 1979, m'est apparu rétrospectivement d'une audace incroyable. Peut-être que le mot

9. Cf. H. Merlin-Kajman, Public et littérature en France au XVII e siècle et «La question de la destination".

Io. Cf. plus haut, note 2 . 
"courage» serait du reste plus adéquat. Dans ces années-là, l'idéologie d'un enseignant de gauche comprenait l'exaltation héroïque de la subversion ou de la transgression; et cela dure encore en plus d'un lieu culturel, du reste. Claude Reichler tourne le dos à une telle naïveté: la subversion va prendre un autre nom, moins... subversif que ne le promet sa bannière, puisqu'elle va se révéler constituer, sur le long terme, un "type de sémioticité antagoniste à l'intérieur d'une culture» ${ }^{11}$. Mais il tourne aussi le dos à la naïveté inverse, celle qui se contenterait de juger du discours séducteur à partir du discours "droit», du discours proprement «sym-bolisant». Son objectif est de montrer que «l'opposition de ces deux modes du dire» - le dire symbolisant, le dire diabolique - est "celle de deux imaginaires investis par les sujets parlants dans le langage, qu'une ambivalence constitutive de celui-ci sécrète» ${ }^{12}$; et, de là, de dégager la poétique du discours séducteur, c'est-à-dire sa double force d'attraction. D'une part, le discours "diabolique» attire le discours "droit» dans sa propre économie, pour le détourner et s'en servir (comme le fait Dom Juan avec le discours de la dévotion), cherchant à montrer par là que le discours droit n'est qu'un cas particulier de discours séducteur. D’autre part, et par voie de conséquence, il exerce aussi son pouvoir d'attraction sur le destinataire qui vit, quant à lui, sous la loi du discours droit et se laisse confondre par les apparences de discours droit que le discours séducteur lui offre en leurre. La diabolie, qui capture l'efficacité sym-bolique pour en inverser la finalité, c'est-à-dire pour disjoindre les cohérences tissées par le symbole, "entraîn[e]" donc "[son] auditeur ou [son] lecteur dans une vrille sans fin ${ }^{13}$.

Or la particularité de La diabolie, c'est d'avoir pris, pour analyser et illustrer cette poétique diabolique, non seulement deux œuvres littéraires, Dom Juan et le Roman de Renart, mais encore une œuvre critique, celle de Roland Barthes. Ce choix, ce trajet, permet à Claude Reichler de dégager l'invisible «logique de l'évolution de la culture française»:

[...] le langage y [est] devenu, sous des prétextes scientifiques, le modèle de toute pratique signifiante (et non seulement le relais ou l'interprétant), et [...] Barthes lui-même [a] aidé à cette promotion en faisant

II. C. Reichler, La diabolie, p. 215 (je souligne).

I2. Ibid., p. 10.

I3. Ibid., p. 16. 
dépendre la sémiologie de la linguistique, pour exercer ensuite d'autant plus sûrement la fonction diabolique en séparant signifié et signifiant ${ }^{14}$.

Et soudain surgit, dans la liste de ces destinataires pris au piège de ce que l'on pourrait appeler leur crédulité symbolique, la première personne du singulier:

[...] il n'y [a] véritablement diabolie que pour un destinataire qui endosse la modélisation sémiotique pervertie par le discours ou par le texte: Charlotte ou Monsieur Dimanche, Ysengrin et Brun, tel étudiant, tel lecteur, celui que j'ai été, et qu'une soif de scientificité, un besoin de sens, portaient à tenir pour vérité un jeu avec les textes et leurs interprétations ${ }^{15}$.

Claude Reichler ajoute:

Mais, en même temps, le texte diabolique explicite son caractère duplice à l'usage d'un destinataire libéré de la sémioticité dans laquelle lui-même est contraint de s'écrire: il met en scène les procédures séductrices, fait la théorie de ses captations, marque l'artifice et le dévoilement, exorcise la fascination qu'il exerce ${ }^{16}$.

Voici sans doute pourquoi il est proprement «littéraire»: la diabolie n'est-elle pas en effet un cas particulier d'interprétation symbolique des modélisations symboliques elles-mêmes - selon la définition proposée ultérieurement par Claude Reichler? Interprétation symbolique «diabolique»? Toute la question est là peut-être: existe-t-il une lecture diabolique d'un texte littéraire, une pratique diabolique de nos disciplines?

Je laisse cette question en suspens et y reviendrai en conclusion. J'entends en tout cas, dans le livre de Claude Reichler, une interpellation: en passant de Dom Juan au Roman de Renart, et de ces deux œuvres à celle de Roland Barthes, en joignant aux victimes de la renardie et de la séduction libertine celles de l'écriture pratiquée et théorisée par Barthes (ou d'une certaine modalité de son écriture), c'est-à-dire en leur ajoutant, concrètement, l'étudiant qu'il a pu être, Claude Reichler nous

I4. Ibid., p. 215.

I5. Ibid., p. 214.

I6. Ibid. 
place devant la question de ce qu'est un enseignement littéraire et de ce que c'est qu'une écriture "critique» non moins que ce que c'est qu'un texte littéraire; bref, de la limite entre la diabolie et la critique.

Cette limite résiderait-elle uniquement, comme certains propos de Claude Reichler pourraient le donner à penser, dans le caractère purement analytique (réflexif, conceptuel, théorique...) de l'activité critique? Ce n'est pas certain. Si je n'ai jamais rencontré de diabolie dans les textes de Claude Reichler, il me semble en revanche pouvoir affirmer qu'il «écrit» aussi, au sens intransitif mais non barthésien du verbe, ce qui signifie qu'il sait jouer de la valeur proprement symbolique (et pas seulement analytique) de ce qu'il écrit : autrement dit, qu'il sait relancer dans son écriture la valeur qu'il assigne aux discours littéraires, à savoir leur fonction d'actualisation symbolique, pour un sujet, des systèmes modélisants eux-mêmes ${ }^{17}$. J'ai ainsi remarqué qu'il ne cite pas toujours les textes qu'il commente: parfois, il les re-raconte. C'est le cas par exemple de l'épisode analysé par Claude Reichler dans «La littérature comme interprétation symbolique» où Saint-Simon raconte la stupeur des courtisans et des militaires découvrant que Louis XIV supervise le siège de Compiègne, parade militaire plus que bataille, penché vers la chaise à porteurs où se tient - qui ? Non pas la reine, une reine, mais $M^{\text {me }}$ de Maintenon. "Cet effet de stupeur, Saint-Simon le "monte" avec un soin tout particulier " ${ }^{18}$, nous dit Claude Reichler. Mais lui aussi : si bien que, tissé dans l'économie symbolique de son propre texte, le commentaire analytique du critique n'en fait ressortir qu'avec plus de force son absence dans le texte du mémorialiste, qui, lui, repose au contraire uniquement sur une interprétation symbolique. En effet, Saint-Simon n'explique pas la cause du scandale: il revoit la scène, la revivifie et la re-marque par l'écriture en en accentuant les éléments saillants et évidemment signifiants. Ecrite, la scène, qui «bafoue la modélisation symbolique " ${ }^{19}$ commune, doit "susciter chez son lecteur une réaction affective et participative, voire une identification au jugement du narrateur et des assistants ${ }^{20}$.

17. «L'interprétation inscrit les procès représentationnels dans la situation, les affects et les visées d'un sujet.»C. Reichler, «La littérature comme interprétation symbolique», p. 98.

I8. Ibid., p. 107.

19. Ibid., p. 108.

2o. Ibid., p. 109. 
L'interprétation analytique de ce texte de Saint-Simon par Claude Reichler ne se sépare donc pas, dans ce cas-ci, de ce que je pourrais appeler son incorporation symbolique. Le mouvement qui porte ainsi l'écriture de Claude Reichler aux frontières de la littérature est constant: qu'on lise par exemple l'extraordinaire mise en scène, à la façon d'un trompe-l'œil baroque, de La confession d'un enfant du siècle, de Musset, qui ouvre La découverte des Alpes et la question du paysage, paru en 2002. Il est encore plus net dans deux textes que je ne commenterai pas mais que je tiens à citer en raison de leur beauté. Le premier, "Une entrevue suivie de lettres», est un texte de "critique-fiction » qui imagine une sorte de rencontre entre Artaud et Rivière ${ }^{21}$. Du second, «Paysages d'origine», je me contenterai de citer une phrase qui suggère qu'une configuration autobiographique singulière informe nos manières de lire: «Un regard existe derrière le mien, qui m'aiguille et m'inquiète. " ${ }^{22}$

Qu'un regard existe derrière le nôtre, qui nous aiguille et nous inquiète, cela pourrait bien constituer non seulement une définition de l'exigence éthique qui nous lie à la culture «lettrée» - tout particulièrement «nous", enseignants et chercheurs -, mais encore l'emblème de ce que la perspective anthropologique ajoute, en termes de décentrement, de perte, de blessure narcissique nécessaire, à ce qui pourrait, sans elle, n'être qu'un simple rapport de tradition continuée, sage voire victorieuse $^{23}$, à ce passé culturel. Résister, sans naïveté ni bien-pensance, à la diabolie n'est pas résister à l'insécurité née de la rencontre avec l'autre,

2I. Paru en 1976 dans le numéro 10-11 de la revue Obliques, consacré à Antonin Artaud. Claude Reichler m'a fait l'amitié et l'honneur d'en autoriser la republication dans la revue en ligne que je dirige, Transitions (http://www.mouvement-transitions. fr/hospitalites/637-une-entrevue-suivie-de-lettres.html). "Le vertige littéraire emporte tout. Ce récit se joue des notions de forme et de conscience, qu'il construit et déconstruit tour à tour, à propos d'une discussion - celle de Rivière et d'Artaud qui porte précisément sur la maîtrise interprétative», écrit Claude Reichler dans le "Postambule» qu'il a rédigé pour cette republication.

22. "Cette expérience, je la revis quand je marche dans les Alpes; je la découvre dans certaines images, et souvent elle m'apprend à lire les textes. Un regard existe derrière le mien, qui m’aiguille et m'inquiète.» C. Reichler, "Paysages d'origine», texte en ligne, sans pagination.

23. Je fais ici allusion à l'affirmation de Benjamin concernant la transmission des biens culturels: "Tous ceux qui à ce jour ont obtenu la victoire, participent à ce cortège triomphal où les maîtres d'autrefois marchent sur les corps de ceux qui aujourd'hui gisent à terre. Le butin, selon l'usage de toujours, est porté dans le cortège. C'est ce qu'on appelle les biens culturels.» W. Benjamin, «Sur le concept d'histoire», p. 433. 
et tout le travail de Claude Reichler sur les récits ethnographiques, de Jean de Brébeuf à Lévi-Strauss en passant par Lafiteau ou Michel Leiris, nous en donne l'exemple. Sa perspective, cependant, ici encore, déplace notre paysage critique. Car elle nous invite à sortir d'une autre alternative, celle qui oppose l'ethnocentrisme au relativisme culturel, en nous suggérant de reconnaître, dans les récits des jésuites en Nouvelle France au XVII siècle par exemple, autre chose qu'une projection, sur l'"autre", de leur propre culture: les jésuites, montre Claude Reichler, traduisent, à destination des lecteurs européens, une culture dans une autre, traduction qui trouve sa première source dans les nécessaires rapports d'interaction culturelle noués au jour le jour entre les missionnaires et les autochtones ${ }^{24}$. On notera que pour Claude Reichler, si un texte littéraire n'est ni discours, ni représentation ni simple modélisation symbolique, c'est parce qu'il organise toujours à la fois un jeu différentiel et une rencontre dont l'inquiétude d'un «regard derrière le mien» me paraît constituer la figure.

Et peut-être un tel regard donne-t-il à celui qui en est enveloppé une lucidité particulière - un regard "devant» lui. Ce qui est sûr, c'est que c'est bien le sentiment d'une sorte de pli du temps qui m'a frappée en relisant les premières lignes de «La littérature comme interprétation symbolique»:

Dans les années soixante, l'étude des textes littéraires constituait un centre d'intérêt fondamental, dont la valeur heuristique et didactique ne faisait de doute pour personne. Les réflexions et les recherches se multipliaient dans un foisonnement et une qualité d'écriture qui firent du commentaire un genre majeur. Plus récemment, notre discipline est apparue comme un champ ravagé. Histoire littéraire, critique, sociologie ou sociocritique, psychanalyse, psychocritique, sémiologie... -, tout cela avait cessé d'être conçu comme les ramifications d'un vaste territoire de la pensée, et ne constituait plus que les parcelles d'un domaine imprécis et mal défendu ${ }^{25}$.

Claude Reichler poursuit par un constat moins pessimiste et l'enchaîne sur une mise en garde:

24. Voir C. Reichler, «Une ethnographie baroque?».

25. C. Reichler, «La littérature comme interprétation symbolique», p. 81. 
Aujourd'hui, les manifestations d'un renouveau d'intérêt pour la littérature et les lettres sont constantes. Pourtant, impossible de revenir simplement aux triomphes de naguère, marqués par une fausse autonomie du littéraire et par une hypertrophie du linguistique. Impossible tout autant d'en appeler aux recettes d'une culture humaniste ou historienne ${ }^{26}$.

Il termine sur une question:

Comment cerner les difficultés que rencontre le praticien des textes? Comment lui rendre à la fois la conscience du caractère irremplaçable de son objet, et la confiance dans l'aspect complexe et mouvant de ses études? Peut-on prendre des divers problèmes qui se posent aux métiers littéraires, une vision qui leur donne un sens, qui permette de les situer réciproquement, de les comparer, et finalement, de construire une perspective unifiante? ${ }^{27}$

Ce diagnostic rendu en 1989 est saisissant. En octobre 2012, j’ai organisé, avec François Cornilliat, professeur de littérature française des XVe et $\mathrm{XVI}^{\mathrm{e}}$ siècles à l'Université de Rutgers, et avec Transitions un colloque intitulé " "Littérature": où allons-nous?" 28 . Le même constat d'un "champ ravagé", les mêmes questions - ou peu s'en faut - formaient notre point de départ et ont trouvé un fort écho chez nos collègues, diagnostic aggravé sans doute par le fait qu'au-delà de nos cercles étroits de spécialistes (de spécialistes de spécialités de plus en plus étroites et séparées), on ne voit guère aujourd'hui de «manifestations d'un renouveau d'intérêt pour la littérature et les lettres", tandis qu'à l'inverse nous assistons de toutes parts à des appels «aux recettes d'une culture humaniste ou historienne».

Que s'est-il passé depuis 1989, qui fait que la discipline ait échoué à "construire une perspective unifiante»? Il faudrait évidemment préciser et affiner ce que je vais avancer de façon trop schématique. Mais, outre une "perte d'assurance» (l'expression est de Zygmunt Bauman ${ }^{29}$ ) propre à la postmodernité - perte d'assurance qui expliquerait sa diabolie? -, bien explicable après les multiples crimes dont la civilisation occidentale

26. Ibid.

27. Ibid.

28. Cf. sa synthèse et sa publication sur le site de Transitions: http://www. mouvement-transitions.fr/hospitalites/542-civilites-qlitteratureq-ou-allons-nous.html.

29. Z. Bauman, La décadence des intellectuels, p. 154. 
s'est révélée coupable, je serais tentée d'incriminer, en tout cas en France, et même si cela paraît contradictoire, l'installation durable de ce que, dans une autre introduction saisissante, celle de son livre L'âge libertin paru en 1987, Claude Reichler a nommé le "pseudo-libertinage»:

Certains aujourd'hui souhaitent un retour du libertinage. Ils voient dans ce mot - dont jamais ils n'élaborent le concept, et dont ils escamotent l'histoire - un souverain remède à nos perplexités. Révolue l'époque des grands systèmes, effacés les dogmes et leurs millénarismes: le temps leur semble ouvert de la légèreté intellectuelle et des frivolités de l'esprit, de l'inconstance et de l'irresponsabilité ${ }^{30}$.

Claude Reichler affirme qu'il faut refuser cette image simpliste du libertinage pour deux raisons. La première, appelons-la analytique: le libertinage historique est tout autre chose que ce pseudo-libertinage, et ce sera l'objet du livre. La seconde est éthique, et, à mon idée, cela signifie qu'elle engage symboliquement la parole universitaire:

Ce dont nous avons besoin ne réside pas dans la déliquescence des attitudes intellectuelles; au contraire, en ce moment de désarroi fécond et de solitude où chacun est renvoyé à ses propres ressources, il faut affirmer la responsabilité de la pensée et pratiquer une éthique du discours $^{31}$.

La suite de ma réflexion va s'inscrire dans le sillage de cette exigence, selon un parcours qui s'adossera à quelques points nodaux de l'œuvre de Claude Reichler.

Et ce parcours va commencer par les Alpes! Ou plutôt par une absence d'Alpes. Il s'agit d'un passage des Mémoires d'Henri de Campion, né en 1613 et mort en 1663. Il se situe en 1644, après que le duc de Beaufort, le «maître» du mémorialiste, a été arrêté parce qu’il est soupçonné, à raison, d'avoir voulu faire assassiner Mazarin. Mais les preuves manquent. Campion, principal témoin à charge en puissance, doit se cacher pour protéger le duc et sa maison. C'est le père de Beaufort, le puissant duc de Vendôme, de sang royal, qui gère la situation et assure le relais du patronage. Les obligations et engagements réciproques concernent notamment les moyens de subsistance de Campion, qui s'exile à Jersey, où il

30. C. Reichler, L'âge libertin, p. 7.

31. Ibid. 
passe deux ans. De son côté, le duc de Vendôme est contraint de sortir de France et se retire d'abord en Italie, puis à Genève, d'où il écrit «une lettre tout à fait obligeante» à Campion, "[l]e priant d'aller passer [s]on exil avec lui» ${ }^{32}$. Malgré ses réticences dues à ce qu'il sait des mœurs du duc de Vendôme, Campion se met en route pour le rejoindre à «SaintGall, en Allemagne, du côté de Constance» où réside le duc. Mais arrivé à Genève, il apprend que ce dernier est parti à Venise, ce qui, dit-il:

[...] me mit dans un extrême embarras, à cause de la longueur du chemin, qui ne pouvait se faire sans péril et grande dépense, ayant quantité de montagnes et de rivières fâcheuses à passer chez différentes nations, parlant la plupart la langue allemande que je n'entendais point, et les autres l'italienne, dont j'avais peu de pratique ${ }^{33}$.

Campion persévère cependant:

J'allai à Lausanne, Morat, Soleure, Baden, Zurich, Coire, passai le dangereux lac de Wallenstat, le Rhin près de sa source, celles du Rhône et du Danube, les Alpes par la Splugen, et entrai dans la Valteline d'où, par Chiavena, j'allai m'embarquer sur le lac de Côme, près le fort de Fuentes, et entrai ensuite dans le Milanais ${ }^{34}$.

Le voyage se poursuit alors en Italie. A Padoue, Campion apprend que le duc est parti pour Rome. Mais à Bologne, on lui dit que ce dernier s'est finalement réfugié à Florence, ce qui réjouit Campion, dit-il :

[...] tant parce que j'étais presque à la fin de mon argent, que fatigué comme un homme qui a été quarante-deux jours à cheval depuis le matin jusqu'au soir, ayant fait [...] quatre cent cinquante lieues sans me reposer qu'un seul jour. Des accidents m'obligèrent d'achever ma route en chevaux de louage, qui m’avaient tellement lassé que, quoique j’aie le corps infatigable, j'étais quasi à bout ${ }^{35}$.

Je n'aurais sans doute pas prêté grande attention à ce passage si ma lecture n'avait pas été médiatisée par celle des textes de Claude Reichler portant sur le voyage dans les Alpes, c'est-à-dire si, moi qui ne suis ni

32. H. de Campion, Mémoires, p. 175.

33. Ibid., p. 179.

34. Ibid., p. 180.

35. Ibid. 
dix-neuviémiste, ni familière de la montagne, je ne m'étais, à travers les analyses de Claude Reichler, approprié «symboliquement» les paysages et si je n'avais aussi, bien sûr, compris "analytiquement" leur importance historique. C'est donc un décalage, perçu à partir d'un point de vue historique postérieur à celui auquel appartient le récit de Campion, qui m’a rendu sensible le quasi-blanc référentiel sur lequel il est construit, et, de là, l'a fait résonner pour moi dans toute son intensité. C'est donc l'«intensité d'une absence» que je voudrais essayer de cerner.

Mais peut-on trouver du sens au fait, trop évident, que manque à Campion la modélisation symbolique permettant d'interpréter les montagnes comme un "paysage» et de s'y représenter lui-même? Ne suis-je pas en train, avec ce fil, de m'engager dans une lecture anachronique qui me condamne au contresens?

Pas exactement, et peut-être même: au contraire.

Une remarque de Claude Reichler éclaire en effet de façon lumineuse le texte de Campion:

[Jusqu'à] la deuxième moitié du XVIII e siècle environ, les processus symboliques étaient fixés sur les liens entre Dieu et le monde, compris d'une manière à la fois analogique, et de parties à totalité. [...] Lorsque cet univers de compréhension se brise, une forme essentielle de modélisation, constitutive de l'équipement mental humain, devient vacante. Mis en présence du monde sans la médiation d'une totalité «régulatrice" [...], l'homme reporte directement sur la nature le mouvement de connaissance symbolique qui transitait auparavant par la divinité. Au lieu de déchiffrer Dieu dans les figures du monde, il va s'y lire luimême, comprendre le monde comme une projection de lui-même [...]. Telle est la finalité du voyage romantique: non d'aller à la rencontre d'une altérité, mais de descendre dans les profondeurs d'un moi qui n'a plus d'image transcendante et que la vie sociale oblitère ${ }^{36}$.

De fait, la relation de symbolisation établie par Campion avec la nature est entièrement médiatisée par Dieu, comme l'indique une phrase qui commente sa décision, prise à Genève, de rejoindre le duc à Venise :

Néanmoins l'habitude de vouloir achever tout ce que je commence me fit résoudre, malgré ces difficultés, à continuer mon voyage, quoique,

36. C. Reichler, «Ecriture et topographie dans le voyage romantique», p. 11. 
par un pressentiment qui ne paraissait pas fondé en apparence, mais que Dieu m’a toujours donné dans les fâcheux accidents qui me sont arrivés, je n'en espérasse nulle satisfaction ${ }^{37}$.

D'une part, la phrase dessine l'ethos du mémorialiste, marqué de constance et de fidélité selon une interprétation symbolique de sa propre conduite et de ses propres pensées conforme aux modélisateurs du stoïcisme chrétien. D’autre part, elle fait allusion à un détail révélant son lien personnel à Dieu, détail sur lequel Campion va revenir à un autre moment clé de ses Mémoires, c'est-à-dire dans le passage qui précède de quelques lignes le récit de la mort de sa petite fille alors âgée de 4 ans. Il séjourne alors dans sa maison:

J'y fus deux mois avec tout sujet de satisfaction, et néanmoins avec une mélancolie qui était le pressentiment de l'extrême affliction qui était prête de m’arriver. Cela m'alarmait, me souvenant que Dieu m'avait toujours averti de mes malheurs par songes, présages, ou sentiments intérieurs. [...] En allant trouver le duc de Vendôme en Italie, une belette me traversa le chemin près de Genève, ce qui m'a toujours été un présage funeste. En arrivant à Rome avec Beaupuis, une autre nous traversa aussi le chemin [...]. Cela m'était aussi arrivé à la prise de Ganseville, où une autre belette me traversa le chemin ${ }^{38}$.

Nous ne saurons rien de ce que Campion a vu ou regardé des Alpes (ni de l'Italie du reste); rien des perceptions esthétiques ${ }^{39}$ qui ont pu être les siennes lors de ce voyage. Nul "paysage» ne l'entoure. Seules les étapes sont mentionnées: "Lausanne, Morat, Soleure, Baden, Zurich, Coire», etc., étapes généralement urbaines qui traduisent l'importance du lien social, de la «conversation». De sa traversée de la Suisse, de son interaction avec la nature, Campion n'a retenu, dans son récit, que deux aspects: d'une part, "quantité de montagnes et de rivières fâcheuses à passer" ou la dangerosité du lac de Wallenstat; d'autre part, une belette qui lui «traverse le chemin». D’évidence, le verbe «traverser» est ici

37. H. de Campion, Mémoires, p. 179.

38. Ibid., p. 211.

39. A la suite de Jacques Rancière, j'entends ici "esthétique» au sens de ce qui concerne le sensible, indépendamment de l'effet causé par les œuvres artistiques liées au «régime esthétique des arts» (J. Rancière, Le partage du sensible, p. 31 sq.). 
d'une extrême importance, il nous permet d'entendre la valeur symbolique accordée à ce qui est pour lui un signe. Un chemin traversé, on le comprend, c'est un sens qui va de travers. Et cette signification donne en retour sa vraie valeur symbolique à la mention de l'épuisement de son corps: les Alpes aussi l'ont "traversé» plus qu'il ne les a traversées.

Ce passage - passage du récit, passage des montagnes - est emblématique du mouvement entier de ses Mémoires: ce qui l'attend à Florence, c'est la trahison du duc de Vendôme qui bafoue la modélisation symbolique censée garantir le lien de service entre les deux hommes. D'où l'information concernant sa fatigue physique: il s'agit là encore de détails dont il faut mesurer l'importance symbolique. Comme Claude Reichler le souligne dans la "Présentation» de l'ouvrage qu'il a dirigé, Le corps et ses fictions, paru en 1983, jusque dans ce qui informe sensations et perceptions, le corps est pris en charge par des «réseaux symboliques» ${ }^{40}$. Campion agit selon la modélisation de la foi donnée, de l'honneur vertueux plus que social, et en montre l'effectuation symbolique et pratique dans son propre corps, dans sa propre expérience, dans ses propres pertes; et ceci vaut preuve.

La traversée des Alpes a coûté de l'argent et de la peine physique à Campion, a mis sa vie en danger: ceci est normal en termes de service, mais mérite reconnaissance et rétribution. Or ses Mémoires montrent le vacillement général des modélisations, si bien que le «moi» de Campion remplit au moins une condition $\mathrm{du}$ "moi» romantique: si ce n'est pas encore un «moi qui n'a plus d'image transcendante», c'est à coup sûr un «moi que la vie sociale oblitère».

Mais en fait, ne serait-ce pas ici que l'anachronisme nous guetterait? Au XVII siècle, il faut plutôt comprendre que c'est cette oblitération sociale qui produit le "moi», en requérant pour lui un lieu - à commencer par un lieu quasi grammatical, la corrélation entre «moi» et «je», pronoms personnels qui trouvent alors leur syntaxe moderne ${ }^{41}$. Souffrant, le moi s'éprouve en attente - en souffrance d'une place. En

40. "Une typologie des images du corps, aussi bien qu'une histoire de sa mise en signes, ou en mots, doivent donc explorer les réseaux symboliques qui le prennent en charge. A cette opération, nulle science ne semble propre: il faut plutôt une métasymbolique capable de faire apparaître en même temps l'objet modélisé et la médiation modélisante, l'articulation qui donne sens et le corps qui trouve une forme. Cette métasymbolique existe: c'est le texte littéraire. " C. Reichler, «Présentation», p. 4.

4I. Cf. T. Cave, Pré-histoires. 
effet, si, outre sa relation personnelle à Dieu, Campion n'est pas sans «image transcendante», c'est qu'il place son écriture sous le signe de trois lectures, réitérées, dit-il, toute sa vie: Plutarque pour «bien vivre», Sénèque pour "bien mourir", et Montaigne pour se "bien connaître» ${ }^{42}$. Ce dernier surtout autorise l'écriture du mémorialiste: l'autoportrait montaignien a "familiarisé" plusieurs générations avec un soi-même "affecté». Et c'est bien cette ressource, la ressource d'un «libertin» qui n'est en rien "dia-bolique», qui soutient Campion dans son interprétation symbolique des conflits de modélisation caractéristiques de son temps et affectant sa propre vie.

La majeure partie du texte est en effet occupée par des récits de guerre. Or il ne s'agit pas pour lui de dresser un portrait héroïque de luimême en suivant l'interprétation symbolique du récit épique. Même si le motif de l'honneur est récurrent, il reste totalement déconnecté d'un souci de gloire. Les sentences sont rares: la vertu, encore une fois, résulte d'un rapport personnel à Dieu et se prouve symboliquement bien plus qu'analytiquement. Et surtout, on ne rencontre aucun passage burlesque.

Dans un livre consacré au métier des armes sous Louis XIV, l'historien Hervé Drévillon a commenté ce qui lui apparaît comme une contradiction. D'un côté, les officiers demandent facilement, de façon très ostentatoire, des rétributions financières pour les services rendus:

Les cicatrices laissées sur le corps formaient un langage censé exprimer la valeur [...]. Dans les placets, par exemple, la douleur n'était pas la conséquence intimement vécue de la blessure, mais l'une de ses manifestations, elle aussi tournée vers le public ${ }^{43}$.

Ici, souffrances et blessures entrent dans "une économie de l'ostentation ${ }^{44}$ : elles disent publiquement le sacrifice consenti, elles mesurent la dette d'honneur contractée par le roi. Malgré la discrétion de Campion, qui ne s'adresse pas au duc de Vendôme, ne lui demande rien, ne lui a rien demandé, nous pouvons rattacher à ce versant public et honorifique ses remarques concernant l'état de son corps à l'issue de son voyage: car c'est le corps en tant qu'il est engagé par un contrat symbolique reconnu, celui du service.

42. H. de Campion, Mémoires, p. 42 sq.

43. H. Drévillon, L'impôt du sang, p. 424 sq.

44. Ibid. 
Mais, poursuit Hervé Drévillon sans cacher son étonnement ni même sa déception, pour lui qui espérait "pénétrer les consciences", entrer dans "l'intimité vécue de la blessure " ${ }^{45}$ en abordant "les formes d'écritures plus tournées vers l'introspection (Mémoires, correspondance) » ${ }^{46}$, ce sont la dérision, le style burlesque qui se révèlent la norme des récits "personnels» quand ils évoquent les souffrances, les maladies, les blessures, les morts. Voici un exemple donné par l'historien. Il s'agit du récit de la bataille de Cassano par un certain Quincy, qui écrit:

Les corps morts nous environnaient si fort, que je passai cette nuit la tête appuyée sur le ventre d'un de ces cadavres. Je dormis parfaitement bien $^{47}$.

Pour Hervé Drévillon, «ces paroles de défi et de dérision prononcées pour conjurer la mort et la douleur constituaient des masques, plutôt que des révélateurs d'une sensibilité " ${ }^{48}$. Mon propre travail m’a amenée à une tout autre conclusion. Je ne crois pas qu'il s'agisse de masque, ni que la contradiction relevée par l'historien en soit une: je crois tout au contraire que cette polarité est révélatrice d'une "sensibilité" très particulière, qui a pour corrélat de forclore l'intime. Au XVII e siècle encore, le rapport au corps est construit, d'une part, selon la modélisation religieuse de la Passion du Christ, outragé et divin, corps moqué et corps en majesté; et, d'autre part, selon la modélisation socio-symbolique du regard extérieur porté sur ce corps, support de dignité sur son versant socio-politique, et d'in-dignité physique sur son versant privé. Bref, la contradiction relevée par Hervé Drévillon entre une présentation publique ostentatoire des blessures et leur présentation privée burlesque appartient aux codes socio-symboliques de l'époque.

Ceci a des conséquences importantes, selon moi, pour notre appréhension des styles littéraires. Nous pourrions, dans certaines limites, nous appuyer sur Bakhtine. Mais un article de Marcel Mauss intitulé "Parentés à plaisanterie» fait mieux ressortir les enjeux anthropologiques de ma lecture. Mauss y met en évidence la présence, dans toute société, même les plus "primitives", d'une organisation binaire des relations

45. Ibid., p. 425.

46. Ibid., p. 426.

47. Cité dans H. Drévillon, L'impôt du sang, p. 426 sq.

48. Ibid., loc. cit. 
interpersonnelles: certaines relations autorisent les plaisanteries voire les brimades ridiculisantes; d'autres sont commandées par le respect ${ }^{49}$. C'est cette dualité qui se joue, selon moi, dans ce qu'Hervé Drévillon observe. Le corps adressé au roi est un corps pris dans la relation à respect, le corps qui concerne la dignité, qui s'inscrit dans les échanges honorifiques. Le corps adressé aux proches est le corps privé, le corps grotesque. Au XVII e siècle encore, tout ce qui concerne la face strictement privée des relations - donc notamment celles qui passent par la communauté du bas corporel - est ordinairement abordé à travers le prisme du burlesque ${ }^{50}$. C'est le cas par exemple chez $\mathrm{M}^{\mathrm{me}}$ de Sévigné, sauf à propos du corps de sa fille, ce qui en souligne l'exception. En revanche, à propos de sa petite fille de 1 an restée à Paris pendant qu'ellemême est en Bretagne, voici ce qu'elle peut écrire: «On me mande que mes petites entrailles se portent bien. Elles vont être habillées; cela est joli, des petites entrailles avec une robe. ${ }^{51}$

Le burlesque, celui des relations extra-textuelles ou celui, esthétiquement intensifié, de la littérature, a pour conséquence (et pour cause) d'inhiber l'empathie, de pétrifier la sensibilité: d'interdire un certain type de modélisation symbolique des liens interindividuels eux-mêmes, lesquels passent inévitablement par le style langagier, les jeux de langage. Plus précisément, lorsque les liens interindividuels sont modélisés soit par les "plaisanteries» soit par les "respects», alors, l'intimité, le partage de cœur à cœur se trouvent sans lieu, tout comme le moi. Sans expression possible, le moi, à la limite, n'existe pas. On n'en mesure que mieux l'importance de la lecture de Montaigne: chez lui, l'interprétation symbolique des modélisations de la personne particulière repose non seulement sur un tout autre ton que celui de l'honneur ou de la gloire,

49. On pourrait mettre ce fait en rapport avec la diabolie. Remarquons en tout cas que le symbolique est donc toujours déjà divisé, polarisé par l'oscillation entre familiarité et honneur (la comédie et la tragédie, le blâme et l'éloge).

50. C'est ce qui explique par exemple que, dans ses propres Mémoires, $\mathrm{M}^{\mathrm{me}}$ de la Guette, strictement contemporaine de Campion et dont le récit est continûment burlesque, puisse délivrer ce court récit d'un épisode tragique, pendant la Fronde: «Cependant le pauvre M. de Chavagnac était poursuivi à outrance; car on voulait ou sa vie ou sa personne. Comme il se sauvait dans les greniers tout nu en chemise, $M^{\mathrm{me}}$ sa femme le voulut suivre toute nue aussi: et étant encore sur la montée, on lui tira quatre ou cinq coups de mousqueton qui la tuèrent sur la place et la firent tomber de haut en bas.» Mme de La Guette, Mémoires, p. 120.

5I. $\mathrm{M}^{\mathrm{me}}$ de Sévigné, lettre du 19 août 1671, dans Correspondance, p. 327. 
ce qui est bien connu; mais, ce qui est moins remarqué, son style n'est jamais burlesque, même si Montaigne l'appelle "comique et privé»: les Essais ouvrent la voie à une toute nouvelle modélisation symbolique de la subjectivation des hommes à partir du XVII e siècle.

Campion en est un exemple remarquable dans sa discrétion. Jamais il ne tombe dans "la convention de la rodomontade" 52 , jamais il ne cherche à faire rire du corps grotesque. Au contraire, il mentionne la pitié qu'il éprouve devant le spectacle des soldats violant des femmes ou brûlant des villes, il réprouve les actes de cruauté, il est attentif à l'affliction qui se peint sur un visage, analyse les nuances des sentiments et des émotions, les siennes comme celles des personnes rencontrées. Enfin, la fin de son récit l'amène même à revendiquer la légitimité des larmes lors de la mort de sa fille de 4 ans, sujet qui pourrait être traité soit sur un mode strictement religieux, soit sur un mode sinon franchement burlesque, du moins dans le sillage de l'économie affective révélée par l'oscillation entre l'honorifique et le burlesque:

Je sais que beaucoup me taxeront de faiblesse, et d'avoir manqué de constance dans un accident qu'ils ne tiendront pas des plus fâcheux; mais à cela je réponds, que les choses ne font effet sur nous, que selon les sentiments que nous en avons, et qu'ainsi il n'en faut pas juger généralement comme si nous avions tous la même pensée. [...] L’on prend souvent l'insensibilité ou la dureté pour de la constance, comme l'amour et l'amitié pour de la faiblesse. J'avoue que je jouerais le personnage d'une femme si j'importunais le monde de mes plaintes; mais chérir toujours ce que j'ai le plus aimé, y penser continuellement en éprouvant le désir de m'y rejoindre, je crois que c'est le sentiment d'un homme qui sait aimer $[\ldots]^{53}$.

On remarquera, cette fois, la prééminence de l'analytique. Mais c'est qu'il s'agit, au point de la plus grande douleur, d'arracher le droit à un changement de repère modélisant. Le mémorialiste affirme ici son droit à «se représenter", à "se publier» dans l'authenticité d'une intériorité affectée. Le ton du passage ressemble à une déclaration: déclaration d'amour paternel, déclaration de chagrin intime et définitif. Prenant à témoin ces «beaucoup» qui le «taxeront de faiblesse», Campion bouleverse l'ordre

52. H. Drévillon, L'impôt du sang, p. 426.

53. H. de Campion, Mémoires, p. 212 sq. 
habituel des sentences, la sémiologie sociale, conventionnelle des émotions, et récuse les modélisateurs attendus pour envisager un nouveau modélisateur symbolique convoqué au bord du désespoir, en direction des hommes et non pas de Dieu, qu'il sait prier par ailleurs.

Déclaration publique, ai-je dit. C'est là que nous arrivons à la question du statut «littéraire" de son texte. Les Mémoires d'Henri de Campion n'étaient pas destinés à la publication ${ }^{54}$. Le mémorialiste, écrit-il, «veu[t] laisser les fruits de $[s]$ on expérience» à ses enfants. De ce but explicite, nous pourrions conclure, suivant les indications de certains critiques et historiens, que ce texte n'a rien d'intrinsèquement "littéraire», que seul un coup de force éditorial ultérieur, soutenu ensuite par les institutions littéraires et des entreprises critiques mystificatrices, peut nous conduire à le lire comme de la littérature; bref, que pour le comprendre, il faut le rendre à sa fonction contextuelle, celle d'une transmission symbolique familiale à valeur éthique et honorifique.

Il est bien vrai que Campion n'est pas un écrivain, si l'on donne à ce mot le sens social qu'Alain Viala lui a donné dans Naissance de l'écrivain. Ce que je voudrais suggérer maintenant, c'est que l'espèce d'anachronisme par lequel je suis passée éclaire quelque chose de l'historicité très particulière de la littérature. Comme l'écrit encore Claude Reichler:

Si l'on abandonne les définitions classiques pour une définition "anthropologique», on comprend mieux le fait que le caractère littéraire d'un texte ne devient opératoire que dans la perception qu'en ont les lecteurs. L'interprétation est le lieu de la subjectivité, et l'on sait qu'un texte peut être jugé littéraire par certains, alors qu'il ne l'est pas pour d'autres ${ }^{55}$.

54. «Si mon dessein était d'écrire pour le public, je choisirais un sujet plus intéressant que celui de ma vie; mais comme ce n'est que pour ma famille et mes amis, je crois que je ne puis rien faire de plus agréable pour eux et de plus commode pour moi, que de leur raconter naïvement les divers événements qui me sont arrivés, en y joignant, pour leur en rendre la lecture plus intéressante et plus profitable, les choses dont j’ai été témoin, tant par rapport aux affaires publiques qu'à celles des particuliers, et qui me sembleront dignes de mémoire. Si je ne puis donner moi-même à mes enfants de bonnes instructions, je veux du moins leur laisser les fruits de mon expérience, ce qui est le seul motif du travail que j'entreprends [...]» (Ibid., p. 39 sq.).

55. C. Reichler, «La littérature comme interprétation symbolique», p. 112. 
Ce propos nous libère de la question de «l'invention de la littérature» ${ }^{56}$. Il se trouve que c'est au terme d'une étrange expérience de lecture que les Mémoires de Campion se sont mis à résonner pour moi. Je les ai lus sans aucune attente littéraire particulière. Je cherchais, pour un livre que je prépare sur la civilité (d'où mon intérêt pour l'honneur et la familiarité), des occurrences de ce terme: je l'ai donc lu comme document. Je ne savais même pas que j'allais y trouver tant de récits de guerre. Au même moment, à l'aide de textes de psychanalystes portant sur l'inscription du trauma dans l'histoire ${ }^{57}$, j'analysais en quoi le zèle guerrier d'Horace, dans Horace de Corneille, s'accompagnait d'une pétrification de la sensibilité, d'une impassibilité devant les liens familiaux ${ }^{58}$.

Ma stupeur, en lisant ces Mémoires de Campion, c'était de découvrir que l'énonciation de ce militaire, qui avait passé une bonne partie de sa vie au milieu de spectacles d'horreur, était "passible» (antonyme d'impassible) et me rendait même sensible à son expérience.

J'aurais pu, face à cette impression de lecture, chercher à la rapporter à une stratégie d'écriture, une rhétorique persuasive. J'aurais pu conclure que le mémorialiste avait réussi son opération d'autoprésentation en caractère vertueux. J'aurais pu, en somme, me diriger vers un «commentaire analytique diabolique» - décidant que le symbolique du mémorialiste n'était qu’un leurre.

Ma démarche a été inverse. Je me suis laissé saisir par son adresse, peut-être parce que, justement, au-delà de l'adresse initiale à ses enfants, un autre geste de donation est présent dans le texte, explicite dans sa déclaration publique de douleur à la mort de sa fille. Ce geste de donation sans destinataire certain vise un temps ultérieur lui-même sans terme particulier: une anachronicité prospective, pourrait-on dire.

56. Cf. F. Dupont, L'invention de la littérature. Je partage avec Claude Reichler une certaine méfiance à l'égard des «inventions", comme il l'écrit à propos de "l'invention des Alpes»: "Ce ne sont pas simplement les Alpes que les voyageurs du XVIII siècle ont découvertes (ils les ont encore moins “inventées", selon une expression dont on abuse), mais une médiance singulière, une imbrication indissociable de nature, de culture, et de subjectivité.» C. Reichler, La découverte des Alpes et la question du paysage, p. 21.

57. Cf. F. Davoine, J.-M. Gaudillière, Histoire et trauma. Cf. aussi P. Loraux, «Les disparus".

58. Sur ces questions, cf. H. Merlin-Kajman, «Peur, rire et outrage» et "Sentir, ressentir». 
A partir de cette sorte d'abandon ou de crédit accordé au texte (c'està-dire de crédit à ma propre réaction "esthétique»), j’ai cherché à comprendre pourquoi j'étais «liée». Ma réponse, je l'ai trouvée dans un paradoxe: je me trouve liée à ce texte par tout ce qu'il ne mobilise pas: pas d'Alpes, pas de complaisance ostentatoire, pas d'epos ni d'héroïsme, pas de corps burlesque, pas de plainte, pas de sentences... Campion minore tous les éléments qui pourraient faire effraction dans la sensibilité du lecteur, comme s'il continuait d'écrire pour sa fille, dont il avait rêvé, dit-il, de se faire plus tard une amie.

La dynamique que je viens de décrire ressemble fort à ce que Claude Reichler écrit du transfert:

Comme l'indique Lévi-Strauss, le transfert mis en évidence par la psychanalyse constitue bien un processus de délégation symbolique. Un énonciateur, acteur d'une situation passée qu'il évoque en la racontant, implique son destinataire actuel (à savoir son psychanalyste) comme protagoniste de la représentation narrée. On vérifie la prégnance des mécanismes d'identification dans ce moment où les distinctions entre présent et passé, entre ici et là-bas, entre énonciateur et énoncé, sont suspendues ${ }^{59}$.

Il faut simplement modifier un mot: «destinataire actuel». L'énonciateur Campion implique un destinataire "inactuel», à venir. Certes, son texte ne "nous" est pas adressé, au sens où il n'est pas destiné à un public. Mais il prend à témoin, et en ce sens, il est bien "adressé». Dans le contexte d'effondrement de son système de modélisation sociosymbolique et de fragilisation extrême que lui cause la mort de sa fille bientôt suivie de celle de sa femme, Campion maintient présent un souvenir, et parle enveloppé par le sentiment que le monde s'est dédoublé; et de ce dédoublement, il fait le moteur d'une adresse - une adresse «symbolique». D'une telle adresse, dès qu'elle trouve un lecteur-interprète qui l'entend et la reçoit, surgit la littérarité.

Revenons du reste une seconde à la belette. Il est remarquable que Campion n'interprète pas ce signe dans le registre de la sorcellerie: la belette n'est pas un signe diabolique mais un signe envoyé par la Providence. Jamais du reste le diable n'apparaît dans ses Mémoires: Campion ne semble en rien concerné par le mythe de la sorcellerie.

59. C. Reichler, «La littérature comme interprétation symbolique», p. 102. 
Claude Reichler range les mythes parmi les interprétations symboliques. Mais le mythe sorcier, en quel sens est-il symbolique? Dans Naming the witch, l'anthropologue James Siegel, contestant sur ce point les analyses de Lévi-Strauss, a récemment montré au contraire son caractère destructeur sur le lien social. Pour arriver à cette conclusion, il est reparti du texte de Lévi-Strauss "Le sorcier et sa magie", qui fait diptyque avec «L'efficacité symbolique» auquel Claude Reichler se réfère pour analyser l'«effet de délégation, propre au dispositif symbolique dans tous ses emplois, de la magie à la poésie et au jeu " ${ }^{60}$ :

Dans une tribu sud-américaine, une parturiente peine; sa vie est menacée, de même que celle de l'enfant. Appelé, le sorcier entame une longue incantation, qui mêle un récit mythique d'exploration et de retour et des gestes rituels. Au bout d'un certain temps, la femme est délivrée ${ }^{61}$.

Et Claude Reichler conclut par un premier rapprochement entre le mythe chamanique et la littérature:

Le symbole peut être en même temps vrai et faux. A ce stade, le récit mythique du sorcier, l'exhibition de la filoche ensanglantée mettent en jeu des phénomènes absolument semblables à ceux qui se produisent lorsque, adolescents, nous lisons «Le bateau ivre», et plus tard, Faust ou tel récit de Conrad... ${ }^{62}$

Dans «Le sorcier et sa magie", Lévi-Strauss fait une analyse analogue. Mais cette fois-ci, il ne s'agit pas d'un récit guérisseur capable d'aider une parturiente à accoucher ou un malade à recouvrer la santé. Il s'agit du récit d'aveu d'un jeune homme zuni accusé d'avoir ensorcelé une fillette atteinte de convulsions. Après avoir nié, le jeune homme zuni se met à raconter ses "méfaits" en une succession de récits de plus en plus détaillés qui fascinent ses juges. Selon Lévi-Strauss, le récit satisfait alors les attentes symboliques du groupe, pour qui un événement était demeuré opaque, élément de réel en mal d'articulation symbolique. La preuve, pour Lévi-Strauss, de la réussite symbolique des récits du jeune homme, c'est que ce dernier n'est pas mis à mort.

6o. Ibid., p. 101.

6I. Ibid.

62. Ibid., p. 102 sq. 
Or James Siegel, en revenant au récit source, relève que le jeune homme n'a dû son salut qu'à l'aide de l'ethnographe, Mathilda CoxeStevenson, qui a rapporté ces faits: si le jeune homme n'a pas été mis à mort, ce n'est pas parce qu'il n'a pas été condamné, c'est parce que Mathilda Coxe-Stevenson l'a aidé à s'enfuir. Selon James Siegel, la mise à mort, la chasse aux sorciers, est le corrélat universel des croyances dans la sorcellerie, des mythes de sorcellerie. Nulle articulation symbolique ici : mais au contraire, un univers de signes qui fonctionnent au compte d'une sorte de destructivité sociale. Le récit du jeune homme zuni, tout efficace, tout fascinant qu'il puisse être, n'articule rien. Il joue son efficacité dans une sémioticité qu'on pourrait appeler diabolique dans le sens que Claude Reichler a donné à ce terme dans La diabolie, alors même que sa séduction répond aux modélisations du groupe: mais ce sont les modélisations du groupe elles-mêmes qui sont, en ce cas, diaboliques, puisqu'elles séparent au lieu de lier.

Or, dernier élément important des analyses de James Siegel: là où il y a mythe de sorcellerie, il n'y a pas d'institution de ce que nous appelons littérature. Il n'y a pas de jeu possible avec ce double énoncé (et cette double énonciation): tu es mort/ je suis mort.

Ce que nous découvre cette analyse, c'est que si les signes lient, ils peuvent lier dans la destruction. Diabolie radicale. Ce n'est pas là le régime de sémioticité de la littérature, du moins la littérature dont j’ai reconnu la scène de destination-donation avec les Mémoires de Campion. En somme, il me semble que si nous devons défendre aujourd'hui la littérature, c'est d'une part en définissant soigneusement nos pratiques - selon moi, elles doivent se garder de la diabolie et s'inscrire, en toute connaissance de cause, dans ce qu'en psychanalyse on nomme "transfert »; et, d'autre part, en circonscrivant les qualités symboliques des textes que nous voulons appeler "littéraires». Comme l'écrit encore Claude Reichler :

$[\mathrm{O}] \mathrm{n}$ voit qu'à partir du moment où la perspective particulière qu'elle ouvre existe (et ce moment peut dépendre d'une manière de lire), la littérature joue dans l'ensemble des représentations fabriquées par une collectivité un rôle imprescriptible. En donnant à connaître les modélisations comme telles, elle trouble les identifications et les positivités, questionne les consensus qui délimitent l'espace commun ${ }^{63}$.

63. Ibid., p. 112 sq. 
Mais elle le fait sans rompre les conditions symboliques, symbolisantes, d'un partage qui n'est ni mythique, ni névrotique, mais qui nous lie de for intérieur à for intérieur. Partage sans lequel, par hypothèse, l'expérience réelle de la démocratie deviendrait difficile...

Hélène Merlin-Kajman

Université de la Sorbonne Nouvelle-Paris III (EA 174)

Directrice du mouvement Transitions

(www.mouvement-transitions.fr) 


\section{BIBLIOGRAPHIE}

Badiou-Monferran, Claire, "Présentation: Le dialogue interdisciplinaire: un "conte de peau d'âne" ?", in Il était une fois l'interdisciplinarité. Approches discursives des Contes de Perrault, dir. par Claire Badiou-Monferran, Louvain-la-Neuve, Academia Bruylant, 2010, p. 5-82.

Bauman, Zygmunt, La décadence des intellectuels. Des législateurs aux interprètes, Paris, Jacqueline Chambon, 2007.

Benjamin, Walter, "Sur le concept d'histoire» (1972), in Euvres, trad. par M. de Gandillac, R. Rochlitz, P. Rusch, Paris, Gallimard, 2000, t. III, p. 429-443.

Campion, Henri de, Mémoires, éd. par Marc Fumaroli, Paris, Mercure de France, 1990.

Cave, Terence, Pré-histoires. Textes troublés au seuil de la modernité, Genève, Droz, 1999.

Davoine, Françoise, Gaudillière, Jean-Max, Histoire et trauma. La folie des guerres, Paris, Stock, 2006.

Drévillon, Hervé, L'impôt du sang. Le métier des armes sous Louis XIV, Paris, Tallandier, 2005.

Dupont, Florence, L'invention de la littérature. De l'ivresse grecque au texte latin, Paris, POL, 1994.

La Guette, $M^{\text {me }}$ de, Mémoires, éd. par Micheline Cuénin, Paris, Mercure de France, 1982.

LÉvi-Strauss, Claude, «L'efficacité symbolique», in Anthropologie structurale, Paris, Plon, 1958, p. 205-226.

—, «Le sorcier et sa magie», in Anthropologie structurale, Paris, Plon, 1958, p. 183-203.

Loraux, Patrice, "Les disparus", in L'art et la mémoire des camps. Représenter. Exterminer, dir. par Jean-Luc Nancy, Paris, Seuil, 2001, p. 41-57.

Mauss, Marcel, "Parentés à plaisanteries», in Essais de sociologie, Paris, Editions de Minuit, 1971, p. 148-161. 
Merlin-Kajman, Hélène, Public et littérature en France au XVII e siècle, Paris, Les Belles Lettres, 1994.

—, "La question de la destination: considérations théoriques", in Eloge de l'adresse, dir. par Anne Chamayou, Arras, Artois Presses Université, 2000, p. 149-161 (Cahiers scientifiques de l'Université d'Artois 14).

—, "Peur, rire et outrage: la face sombre de la "culture carnavalesque" ", in Peur et littérature du Moyen Age au XVII e siècle, éd. par Pascal Debailly, Florence Dumora, Textuel, 51 (2007), p. 169-189.

—, "Sentir, ressentir: émotion privée, langage public", in Les émotions publiques et leur langage au XVII e siècle, dir. par Hélène MerlinKajman, Littératures classiques, 68 (2009), p. 335-354.

RanCière, Jacques, Le partage du sensible, Paris, La Fabrique, 2008.

Reichler, Claude, La diabolie. La séduction, la renardie, l'écriture, Paris, Editions de Minuit, 1979.

—, "Présentation", in Le corps et ses fictions, dir. par Claude Reichler, Paris, Editions de Minuit, 1983, p. 1-5.

—, L'âge libertin, Paris, Editions de Minuit, 1987.

-, "Avant-propos", in L'interprétation des textes, dir. par Claude Reichler, Paris, Editions de Minuit, 1989, p. 1-8.

—, "La littérature comme interprétation symbolique», in L'interprétation des textes, dir. par Claude Reichler, Paris, Editions de Minuit, 1989, p. 81-113.

—, «Les stances devant la mort dans le théâtre baroque ou le retrait du sage», Littérature, 75 (1989), p. 79-91.

—, «Ecriture et topographie dans le voyage romantique: la figure du gouffre", Romantisme, 69 (1990/3), p. 3-13.

—, La découverte des Alpes et la question du paysage, Lausanne, Georg, 2002.

—, «Paysages d'origine», L'Alpe, 43 (2009), texte en ligne (sans pagination), http://www.lalpe.com/lalpe-43-montagnes-intimes/claudereichler.

—, «Une entrevue suivie de lettres» (1976), Transitions, (2011), texte en ligne (sans pagination), http://www.mouvement-transitions.fr/ hospitalites/637-une-entrevue-suivie-de-lettres.html.

—, "Une ethnographie baroque?", in Représentation, métissage et pouvoir. La dynamique coloniale des échanges entre Autochtones, Européens et Canadiens (XVI ${ }^{e}-X X^{e}$ siècle), dir. par Alain Beaulieu, 
Stéphanie Schaffray, Laval, Presses de l'Université de Laval, 2012, p. 319-333.

SÉvigné, $M^{\text {me }}$ de, Correspondance, tome I, mars 1646-juillet 1675, éd. par Roger Duchêne, Paris, Gallimard, 1972.

Siegel, James, Naming the witch, Standford, Standford University Press, 2006.

VIala, Alain, Naissance de l'écrivain. Sociologie de la littérature à l'âge classique, Paris, Editions de Minuit, 1985. 